\title{
Second Human Pegivirus in Hepatitis C Virus-Infected and Hepatitis C Virus/HIV-1- Co-infected Persons Who Inject Drugs, China
}

\section{Haiying Wang, ${ }^{1}$ Zhengwei Wan, ${ }^{1}$ Qiang Sun, ${ }^{1}$ Nalin Zhu, Tianyi Li, Xuqi Ren, Xiaoping An, Shuyun Deng, Yue Wu, Xiufen Li, Lin Li, Jingyun Li, Yigang Tong, Shixing Tang}

We report the presence of the second human pegivirus (HPgV-2) in Guangdong and Sichuan Provinces in China. The prevalence of HPgV-2 in hepatitis C virus/HIV-1-co-infected persons who inject drugs was $12.9 \%$ in Guangdong and $15.9 \%$ in Sichuan. This population is at high risk for HPgV-2 infection.

$\mathrm{I}^{\mathrm{n}}$ n 2015, the second human pegivirus ( $\mathrm{HPgV}-2)$ was inde1 pendently reported by 2 groups in the United States $(1,2)$. Previous reports have indicated that $\mathrm{HPgV}-2$ (also known as $\mathrm{HHpgV-1)}$ is a transfusion-transmitted virus and is associated with hepatitis $\mathrm{C}$ virus $(\mathrm{HCV})$ infection $(1-5)$. The distribution and prevalence of $\mathrm{HPgV}-2$ infection worldwide are of great importance but remain to be determined. In this study, we demonstrate the existence of $\mathrm{HPgV}-2$ in the southern province of Guangdong and southwestern province of Sichuan in China. We have also identified HCVinfected persons, in particular HCV/HIV-1 co-infected persons who inject drugs (PWID), as populations at high risk for $\mathrm{HPgV}-2$ infection. In addition, our work reveals the difference in the prevalence, distribution, and phylogeny between the first human pegivirus ( $\mathrm{HPgV}$; formerly GB virus $\mathrm{C}$ or hepatitis $\mathrm{G}$ virus) $(6,7)$ and $\mathrm{HPgV}-2$.

\section{The Study}

In our initial investigation of $\mathrm{HPgV}-2$, we screened a total of 367 delinked serum or plasma samples from high-risk groups for infection with HCV and HIV-1 and 500 healthy volunteer blood donors from Guangdong Province, China, by using ELISA $(2,5)$, and a nested reverse transcription

Author affiliations: Southern Medical University, Guangzhou, China (H. Wang, Z. Wan, N. Zhu, Y. Wu, X. Li, S. Tang); Guangdong Provincial Key Laboratory of Tropical Disease Research, Guangzhou (H. Wang, Z. Wan, N. Zhu, Y. Wu, X. Li, S. Tang); Beijing Institute of Microbiology and Epidemiology, Beijing, China (Q. Sun, T. Li, X. An, L. Li, J. Li, Y. Tong); Guangdong Provincial Dermatology Hospital, Guangzhou (X. Ren); Nanfang Hospital, Guangzhou (S. Deng)
PCR targeting both the $5^{\prime}$ untranslated region and nonstructural protein 3 regions of $\mathrm{HPgV}-2(3,5)$. We observed a low frequency $(0.4 \%)$ of HPgV-2 antibody detection and the absence of $\mathrm{HPgV}-2$ viremia in healthy blood donors tested in our study. Out of $86 \mathrm{HCV}$-infected patients, 1 (1.2\%) was positive for both $\mathrm{HPgV}-2$ antibodies and viral RNA (Table 1). Furthermore, we did not detect HPgV-2 RNA in men who have sex with men (MSM), although $1(0.5 \%)$ of the $211 \mathrm{MSM}$ was weakly positive for HPgV-2 antibodies and negative for HPgV-2 RNA (Table 1; Figure 1).

We observed a relatively high prevalence of $\mathrm{HPgV}$ 2 infection in HCV/HIV-1 co-infected PWID in Guangdong Province; $12.9 \%$ (9/70) were positive for $\mathrm{HPgV}-2$ antibodies and 5.7\% (4/70) for HPgV-2 RNA (Table 1). We obtained similar results from 270 PWID from Sichuan Province; $15.9 \%$ (43/270) were positive for HPgV2 antibodies and 3.0\% (8/270) for HPgV-2 RNA (Table 1). Using the Fisher exact test, we observed a statistically significant difference between $\mathrm{HCV}$-positive and HCVnegative patients in the prevalence of having $\mathrm{HPgV}-2$ antibodies $(6.2 \%$ vs. $0 ; p<0.001)$ and prevalence of having HPgV-2 RNA (5\% vs. 0; $\mathrm{p}=0.026$ ). Similarly, we observed a statistically significant difference between HIV1-positive/HCV-positive patients and HIV-1-positive/ $\mathrm{HCV}$-negative patients in the prevalence of having $\mathrm{HPgV}$ 2 antibodies $(10 \%$ vs. $0 ; p<0.001)$ and prevalence of having HPgV-2 RNA ( $4 \%$ vs. $0 ; p=0.040$ ) (Table 2). These findings indicate a close association between $\mathrm{HPgV}-2$ and $\mathrm{HCV}$ infection and synergy between HIV-1 and HCV infection with respect to $\mathrm{HPgV}-2$ infections (5).

Furthermore, we obtained 6 near full-length genome sequences of $\mathrm{HPgV}-2$ by using next-generation sequencing or sequencing of PCR products (5). These strains from China, which included 2 from PWID (IDU31 and SC-LS-01), 2 from HCV-infected patients (HCV-121 and C346), and 2 from HCV-infected blood donors (HCV1241 and HCV1563), exhibited an identity of $93.6 \%-97.8 \%$ at the whole-genome level. Compared with other HPgV-2 strains from the United States and United Kingdom, the nucleotide sequence identity was $93.7 \%-96.2 \%$. Sequence divergence was greatest at synonymous sites, with ratios of nonsynonymous to synonymous nucleotide substitutions of $0.125-0.150$, which are consistent with 
Second Human Pegivirus, China

Table 1. Detection frequencies of HPgV-2 in different populations in Guangdong and Sichuan Provinces, China*

\begin{tabular}{|c|c|c|c|c|}
\hline \multirow[b]{2}{*}{ Province, group, and subgroup } & \multirow[b]{2}{*}{ No. tested } & \multicolumn{2}{|c|}{ HPgV-2 } & \multirow[b]{2}{*}{ HPgV RNA+, no. (\%) } \\
\hline & & $\mathrm{Ab}+$, no. $(\%)$ & RNA+, no. (\%) & \\
\hline \multicolumn{5}{|l|}{ Guangdong Province } \\
\hline \multicolumn{5}{|l|}{ HCV-infected patients } \\
\hline $\mathrm{Ab}+/ \mathrm{RNA}+$ & 57 & $1(1.8)$ & $1(1.8)$ & $8(14.0)$ \\
\hline $\mathrm{Ab}-/ \mathrm{RNA}+$ & 7 & 0 & 0 & 0 \\
\hline$A b+/ R N A-$ & 22 & 0 & 0 & $4(18.2)$ \\
\hline Total & 86 & $1(1.2)$ & $1(1.2)$ & $12(14.0)$ \\
\hline \multicolumn{5}{|l|}{ PWID } \\
\hline HIV-1+/HCV Ab+/RNA+ & 70 & $9(12.9)$ & $4(5.7)$ & $28(40.0)$ \\
\hline \multicolumn{5}{|l|}{ MSM } \\
\hline HIV-1+/HCV+ & 12 & $1(8.3)$ & 0 & $4(33.3)$ \\
\hline HIV-1+/HCV- & 100 & 0 & 0 & $28(28.0)$ \\
\hline HIV-1-/HCV+ & 10 & 0 & 0 & $1(10.0)$ \\
\hline HIV-1-/HCV- & 89 & 0 & 0 & $7(7.9)$ \\
\hline Total & 211 & $1(0.5)$ & 0 & $40(19.0)$ \\
\hline \multicolumn{5}{|l|}{ Blood donors } \\
\hline HIV-1-/HCV-/HBV- & 500 & $2(0.4)$ & 0 & NT \\
\hline HCV Ab+/HCV RNA+ & 2 & 2 & 2 & NT \\
\hline \multicolumn{5}{|l|}{ Sichuan Province } \\
\hline \multicolumn{5}{|l|}{ PWID } \\
\hline $\mathrm{HIV}-1+/ \mathrm{HCV} \mathrm{Ab+}$ & 270 & $43(15.9)$ & $8(3.0)$ & NT \\
\hline
\end{tabular}
men; NT, not tested; PWID, people who inject drugs; +, positive; - , negative.

other reports (1-3). Phylogenetic analysis indicated that $\mathrm{HPgV}-2$ strains from China, the United States, and the United Kingdom clustered together to form a separate branch and fell into group 1 with the closely related pegiviruses from bats and rodents (Figure 2). Other pegiviruses from human, simian, and equine sources formed group 2 , in which the variants of $\mathrm{HPgV}$ fell into a separate clade. These results illustrate the difference between the 2 human pegiviruses $(1,2,8)$ and the low level of genetic diversity of $\mathrm{HPgV}-2$ strains (1-3).

In contrast to our findings on $\mathrm{HPgV}-2$ infection, we observed a high frequency of $\mathrm{HPgV}$ infection across all 3 populations tested (HCV-infected patients, PWID, and MSM) (Tables 1, 2). The percentage of $\mathrm{HPgV}$ viremia was $14.0 \%(14 / 86)$ in $\mathrm{HCV}$-infected patients, $19.0 \%$ (40/211) in MSM, and 40.0\% (28/70) in PWID (Table 1). Among MSM, the prevalence of HPgV RNA was $28.0 \%(28 / 100)$ in those who were infected with HIV-1 alone and $33.3 \%(4 / 12)$ in those who were HIV-1/HCV co-infected (Table 1). For MSM who were negative for both HIV-1 and HCV, 7.9\% (7/89) were positive for HPgV RNA (Table 1).

\section{Conclusions}

We report the detection of the second human pegivirus, $\mathrm{HPgV}-2$, in HCV-infected (in particular HCV/HIV-1 coinfected) persons in Guangdong and Sichuan Provinces, China (Table 1). Our results and those from previous studies demonstrate that the virus occurs in several geographically distinct regions in the world $(1-4,9,10)$.

$\mathrm{HPgV}$ and $\mathrm{HPgV}-2$ are the only known human pegiviruses (8), and comparing their association with $\mathrm{HCV}$ and HIV-1 infection is of great interest. Consistent with previous reports, we found that the prevalence of $\mathrm{HPgV}$ viremia was $7.9 \%$ in $\mathrm{HCV}$ and HIV-1-negative MSM and $33.3 \%-40 \%$ in $\mathrm{HCV} / \mathrm{HIV}-1$ co-infected MSM and PWID (Table 1 ). In contrast, only $0.5 \%$ of MSM and $0.4 \%$ of healthy blood donors were positive for HPgV-2 antibodies, but all were negative for HPgV-2 RNA (Table 1). These results indicate that $\mathrm{HPgV}-2$ infection might be much less frequent than $\mathrm{HPgV}$ infection, possibly because of its low transmissibility or high clearance rate (2-4). The dramatic difference of distribution and prevalence between $\mathrm{HPgV}$ and $\mathrm{HPgV}-2$ infections in different populations provides a clue for investigation of disease association with $\mathrm{HPgV}$ 2. $\mathrm{HPgV}$ does not cause human diseases (11) and can inhibit HIV-1 replication as well as prolong survival of

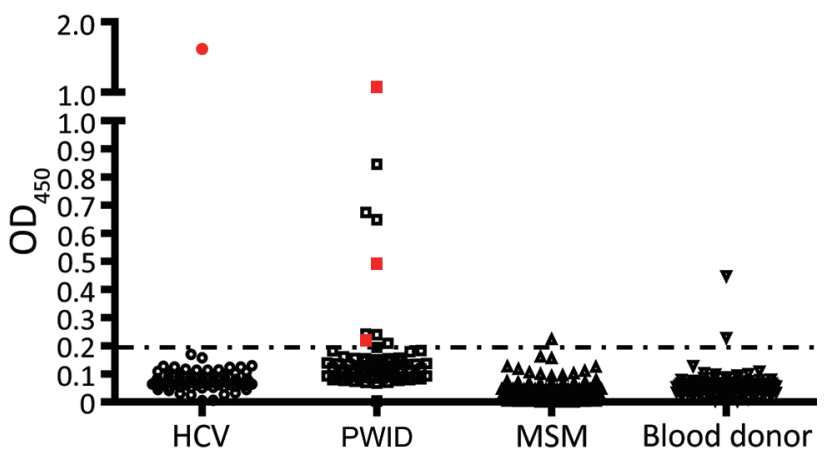

Figure 1. Detection of second human pegivirus (HPgV-2) antibodies in different samples in Guangdong and Sichuan Provinces, China. Serum or plasma samples from $86 \mathrm{HCV}$ infected patients, 70 PWID, 122 MSM, and 102 blood donors (100 samples that were negative for HPgV-2 antibodies plus 2 positive samples) are included. The antibody titers from each sample are plotted on the $y$-axis. HPgV-2 RNA-positive samples are shown in red. $\mathrm{HCV}$, hepatitis $\mathrm{C}$ virus; MSM, men who have sex with men; $\mathrm{OD}_{450}$, optical density at $450 \mathrm{~nm}$; PWID, persons who inject drugs. 
Table 2. Comparison of HPgV and HPgV-2 infections among HCV-, HIV-1-, and HIV-1/HCV-infected populations in Guangdong Province, China*

\begin{tabular}{|c|c|c|c|c|c|c|c|}
\hline \multirow[b]{2}{*}{ Group } & \multirow[b]{2}{*}{ No. tested } & \multicolumn{2}{|c|}{ HPgV-2 Ab+ } & \multicolumn{2}{|c|}{ HPgV-2 RNA+ } & \multicolumn{2}{|c|}{ HPgV RNA+ } \\
\hline & & No. (\%) & $p$ value & No. (\%) & $p$ value & No. (\%) & $p$ value \\
\hline \multicolumn{8}{|l|}{$\mathrm{HCV}$} \\
\hline+ & 178 & $11(6.2)$ & $<0.001$ & $5(2.8)$ & 0.026 & $45(25.3)$ & 0.130 \\
\hline- & 189 & $0(0)$ & & $0(0)^{\prime}$ & & 35 (18.5) & \\
\hline \multicolumn{8}{|l|}{ HIV-1 } \\
\hline+ & 182 & $10(5.5)$ & 0.005 & $4(2.2)$ & 0.212 & $60(33.0)$ & $<0.001$ \\
\hline - & 185 & $1(0.5)$ & & $1(0.5)$ & & $20(10.8)$ & \\
\hline \multicolumn{8}{|c|}{ HIV-1/HCV } \\
\hline$+/+$ & 82 & $10(12.2)$ & $<0.001$ & $4(4.9)$ & 0.040 & $32(39.0)$ & 0.154 \\
\hline$+/-$ & 100 & $0(0)$ & & $0(0)$ & & $28(28.0)$ & \\
\hline
\end{tabular}

HIV-1-infected and Ebola virus-infected patients (12-14). However, possible pathogenicity and disease association of $\mathrm{HPgV}-2$ remain to be elucidated.

The high-risk populations susceptible to $\mathrm{HPgV}-2$ infection includes HCV-infected patients and, in particular, HCV/HIV-1 co-infected PWID. Most (93.3\%) of HPgV2 infected patients were also co-infected with HCV (1-4). Notably, the relatively high frequency of HPgV-2 RNA detection was observed in HCV/HIV-1 co-infected PWID in Guangdong (5.7\%) and Sichuan (3.0\%) Provinces of China (Table 1) and in the United States (10.9\%) (9). In contrast, a somewhat lower percentage (1.7\%) of HCV-positive PWID in the United Kingdom were reported to be HPgV-2 RNA positive, whereas none of the $30 \mathrm{HIV}-1$ singly infected and
$36 \mathrm{HCV} / \mathrm{HIV}-1$ co-infected PWID were positive for HPgV2 RNA (3). These discordant results warrant more studies in different countries to address the association between $\mathrm{HPgV}-2$ and $\mathrm{HCV} / \mathrm{HIV}-1$ co-infection.

Our findings are subject to 2 limitations. First, because a limited number of samples from only 2 provinces of China were tested, the results might not represent overall prevalence of HPgV-2 infection throughout all of China. Second, this study was a cross-sectional rather than a longitudinal study, therefore, the proportions of persistent infection and natural history of $\mathrm{HPgV}-2$ infection remain to be determined.

Future studies should address several questions: whether the close association between $\mathrm{HPgV}-2$ and $\mathrm{HCV}$ infection represents a biologic dependence of these 2

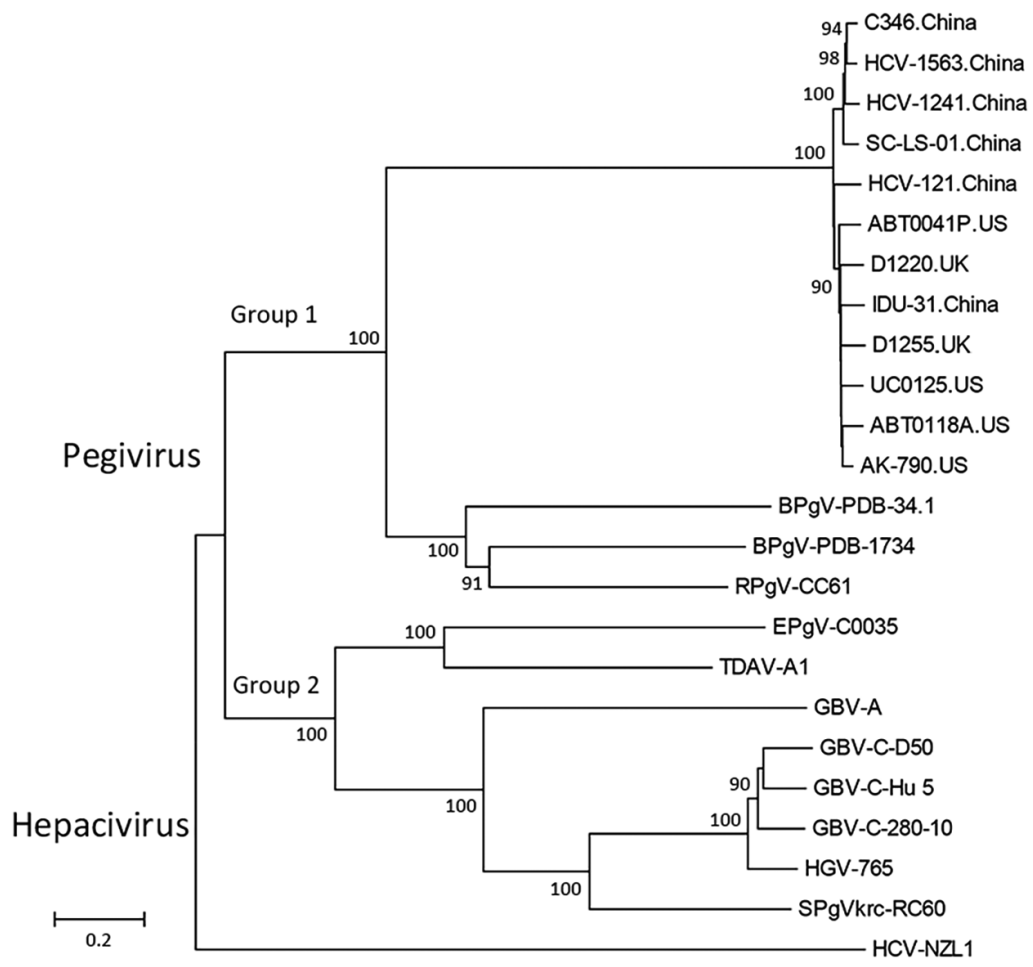

Figure 2. Phylogenetic analysis of second human pegivirus (HPgV2) isolates identified in our study (China) and abroad (UK and US). Phylogenetic trees of nucleotide sequences from complete sequences of HPgV-2 strains isolated in our study and elsewhere as well as hepatitis $C$ virus and pegivirus strains from humans, simians, equids, bats, and rodents are included. The phylogenetic trees were constructed with the neighbor-joining tree method using MEGA6 software (http:// www.megasoftware.net). Bootstrap analysis with 1,000 replicates was performed to determine the robustness of branching; values are shown on branches. Scale bar indicates the estimated number of nucleotide substitutions per site. The near full-length genome sequences of HPgV-2 identified in this study

Human have been submitted to GenBank under accession numbers KX528230 (HCV-121), KX528231 (IDU31),

I Simian KY971606 (C346), MG457178 (SC-

I Human LS-01), MF770985 (HCV1241), and MF770986 (HCV1563). UK, United Kingdom; US, United States. 
viruses; how $\mathrm{HCV} / \mathrm{HIV}-1$ co-infection facilitates $\mathrm{HPgV}$ 2 infection; and whether HCV or HIV-1 viral proteins enhance the transmissibility or infectivity of HPgV-2. In addition, because the rarity of $\mathrm{HPgV}-2$ detection in MSM could be a result of the low frequency of $\mathrm{HCV}$ or HIV-1 infection or the transmission route of HPgV-2, further research should aim to determine if $\mathrm{HPgV}-2$ is more like a transfusion-transmitted virus rather than a sexually transmitted virus.

This work was supported by the Bureau of Science and Information Technology of Guangzhou Municipality (grant nos. 201604020011, 2014Y2-00550, and 201704020219) and the Beijing Municipal Science and Technology Project (grant no. D141100000314001).

\section{About the Author}

Dr. Wang is a postdoctoral fellow at the School of Public Health of Southern Medical University in Guangzhou. Her research interests include identification and diagnosis of viral pathogens and investigation of viral pathogenesis.

\section{References}

1. Kapoor A, Kumar A, Simmonds P, Bhuva N, Singh Chauhan L, Lee B, et al. Virome analysis of transfusion recipients reveals a novel human virus that shares genomic features with hepaciviruses and pegiviruses. MBio. 2015;6:e1466-15. http://dx.doi.org/10.1128/mBio.01466-15

2. Berg MG, Lee D, Coller K, Frankel M, Aronsohn A, Cheng K, et al. Discovery of a novel human pegivirus in blood associated with hepatitis C virus co-infection. PLoS Pathog. 2015;11:e1005325. http://dx.doi.org/10.1371/journal.ppat.1005325

3. Bonsall D, Gregory WF, Ip CL, Donfield S, Iles J, Ansari MA, et al. Evaluation of viremia frequencies of a novel human pegivirus by using bioinformatic screening and PCR. Emerg Infect Dis. 2016;22:671-8. http://dx.doi.org/10.3201/ eid2204.151812

4. Coller KE, Berg MG, Frankel M, Forberg K, Surani R, Chiu CY, et al. Antibodies to the novel human pegivirus 2 are associated with active and resolved infections. J Clin Microbiol. 2016;54:2023-30. http://dx.doi.org/10.1128/JCM.00515-16
5. Wang H, Wan Z, Xu R, Guan Y, Zhu N, Li J, et al. A novel human pegivirus, $\mathrm{HPgV}-2$ (HHpgV-1), is tightly associated with hepatitis $\mathrm{C}$ virus (HCV) infection and $\mathrm{HCV} /$ human immunodeficiency virus type 1 coinfection. Clin Infect Dis. 2018; 66:29-35. http://dx.doi.org/10.1093/cid/cix748

6. Simons JN, Leary TP, Dawson GJ, Pilot-Matias TJ, Muerhoff AS, Schlauder GG, et al. Isolation of novel virus-like sequences associated with human hepatitis. Nat Med. 1995;1:564-9. http://dx.doi.org/10.1038/nm0695-564

7. Linnen J, Wages J Jr, Zhang-Keck ZY, Fry KE, Krawczynski KZ, Alter H, et al. Molecular cloning and disease association of hepatitis $\mathrm{G}$ virus: a transfusion-transmissible agent. Science. 1996;271:505-8. http://dx.doi.org/10.1126/ science.271.5248.505

8. Smith DB, Becher P, Bukh J, Gould EA, Meyers G, Monath T, et al. Proposed update to the taxonomy of the genera Hepacivirus and Pegivirus within the Flaviviridae family. J Gen Virol. 2016;97:2894-907. http://dx.doi.org/10.1099/jgv.0.000612

9. Kandathil AJ, Breitwieser FP, Sachithanandham J, Robinson M, Mehta SH, Timp W, et al. Presence of human hepegivirus-1 in a cohort of people who inject drugs. Ann Intern Med. 2017;167:1-7. http://dx.doi.org/10.7326/M17-0085

10. Frankel M, Forberg K, Coller KE, Berg MG, Hackett J Jr, Cloherty G, et al. Development of a high-throughput multiplexed real-time RT-PCR assay for detection of human pegivirus 1 and 2. J Virol Methods. 2017;241:34-40. http://dx.doi.org/10.1016/ j.jviromet.2016.12.013

11. Mohr EL, Stapleton JT. GB virus type C interactions with HIV: the role of envelope glycoproteins. J Viral Hepat. 2009;16:757-68. http://dx.doi.org/10.1111/j.1365-2893.2009.01194.x

12. Vahidnia F, Petersen M, Stapleton JT, Rutherford GW, Busch M, Custer B. Acquisition of GB virus type $\mathrm{C}$ and lower mortality in patients with advanced HIV disease. Clin Infect Dis. 2012 ;55:1012-9. http://dx.doi.org/10.1093/cid/cis589

13. Zhang W, Chaloner K, Tillmann HL, Williams CF, Stapleton JT. Effect of early and late GB virus $\mathrm{C}$ viraemia on survival of HIV-infected individuals: a meta-analysis. HIV Med. 2006;7:173-80. http://dx.doi.org/10.1111/ j.1468-1293.2006.00366.x

14. Lauck M, Bailey AL, Andersen KG, Goldberg TL, Sabeti PC, O'Connor DH. GB virus C coinfections in west African Ebola patients. J Virol. 2015;89:2425-9. http://dx.doi.org/10.1128/ JVI.02752-14

Address for correspondence: Shixing Tang, Southern Medical University, School of Public Health, 1838 N Guangzhou Ave, Guangzhou, 510515, China; email: tamgshixing@smu.edu.cn 